\title{
Constraints on the early-stage rupture process of the 2011 Tohoku-oki earthquake from 1-Hz GPS data
}

\author{
Yukitoshi Fukahata $^{1 *}$, Yuji Yagi ${ }^{2}$, and Shin'ichi Miyazaki ${ }^{3}$ \\ ${ }^{1}$ Disaster Prevention Research Institute, Kyoto University, Kyoto, Japan \\ ${ }^{2}$ Graduate School of Life and Environmental Sciences, University of Tsukuba, Ibaraki, Japan \\ ${ }^{3}$ Graduate School of Science, Kyoto University, Kyoto, Japan
}

(Received December 27, 2011; Revised September 8, 2012; Accepted September 20, 2012; Online published January 28, 2013)

\begin{abstract}
From the comparison of observed 1-Hz GPS data with simple forward computation that evaluates near and intermediate field terms, we put constraints on the early-stage rupture process of the 2011 Tohoku-oki earthquake. Mainly based on the time difference of the onset of large eastward displacements at stations along the northern coast of the source region, we estimate that the first significant moment release started around $35 \mathrm{~km}$ west of the hypocenter determined by JMA. The moment release occurred approximately from $20 \mathrm{~s}$ to $35 \mathrm{~s}$ after the initiation of the earthquake. Significant moment release around the hypocenter and in the near-trench region started from 25 s, and $35 \mathrm{~s}$, at the earliest, respectively. Before about $20 \mathrm{~s}$, the moment release of the 2011 Tohoku-oki earthquake must have been so small that any substantial displacements were not observed on land. The clear opposite motion that follows the large eastward displacement observed at many stations is due to the intermediate $S$-wave term. Key words: The 2011 Tohoku-oki earthquake, 1-Hz GPS, rupture process, near field term, intermediate $S$-wave term.
\end{abstract}

\section{Introduction}

In order to understand the 2011 Tohoku-oki earthquake, it is crucially important to clarify the rupture process. Especially, the early-stage rupture process probably holds the principal key as to why the earthquake grew as large as magnitude 9. Many rupture models have been proposed for this earthquake mainly from seismic waveform inversion. These results have some common features, such as very large slip near the trench around 60 to $80 \mathrm{~s}$ (Ide et al., 2011; Lee et al., 2011; Shao et al., 2011; Suzuki et al., 2011; Yagi and Fukahata, 2011; Yoshida, K. et al., 2011; Yoshida, Y. et al., 2011; Yue and Lay, 2011) as well as the form of the moment-rate function, which has a shoulder around 35 s, a large peak at about $70 \mathrm{~s}$, and which decreases to zero roughly at $150 \mathrm{~s}$ (Ammon et al., 2011; Hayes, 2011; Lay et al., 2011; Lee et al., 2011; Shao et al., 2011; Suzuki et al., 2011; Yagi and Fukahata, 2011; Yoshida, Y. et al., 2011; Yue and Lay, 2011), where time is measured from the initiation of the earthquake. However, the results of seismic waveform inversion show a significant difference in the rupture process during the initial 30 or $40 \mathrm{~s}$. In some analyses, the rupture continues around the hypocenter (Shao et al., 2011; Yoshida, K. et al., 2011; Yoshida, Y. et al., 2011), whereas, in other analyses, large moment release occurs only in the down-dip (Ide et al., 2011; Koketsu et al.,

\footnotetext{
*Now at: Department of Earth Sciences, University of Oxford, UK.
}

Copyright (C) The Society of Geomagnetism and Earth, Planetary and Space Sciences (SGEPSS); The Seismological Society of Japan; The Volcanological Society of Japan; The Geodetic Society of Japan; The Japanese Society for Planetary Sciences; TERRAPUB.

doi:10.5047/eps.2012.09.007
2011), up-dip (Lee et al., 2011), or both regions (Yagi and Fukahata, 2011).

Other than seismic waveform inversion, a back projection analysis, using the Metropolitan Seismic Observation Network (MeSO-net) that passes through the Tokyo Metropolitan area, shows moment release both in the down-dip and up-dip regions (Honda et al., 2011). The MeSO-net data is considered to have a better resolution for the across-arc direction rather than the along-arc direction, because of the spatial relation between the network and the source area of the earthquake. The generation area of the first strong motion was commonly estimated in the down-dip region from the hypocenter based on the analysis of near-field strong motion data (Asano and Iwata, 2012; Suzuki et al., 2011; Yoshida, Y. et al., 2011). However, the strong motion data have defects in that they are limited in resolution in the across-arc direction, and their amplitude can be larger as a result of fast slip-rate change rather than large slip itself.

1-Hz GPS data observed by the Geospatial Information Authority (GSI) can give important constraints on this problem. Because GPS data record displacements, even if the sampling rate is high, the GPS data are more directly related with the moment release during the rupture process. In this paper, by comparing simple forward computation with observed 1-Hz GPS data, we aim to clarify the early stage rupture process of the 2011 Tohoku-oki earthquake.

\section{1-Hz GPS Data}

Time series of surface displacements are recorded by the GPS Earth Observation Network (GEONET), operated by the Geospatial Information Authority (GSI) of Japan. GPS phase data with a sampling interval of one second are 


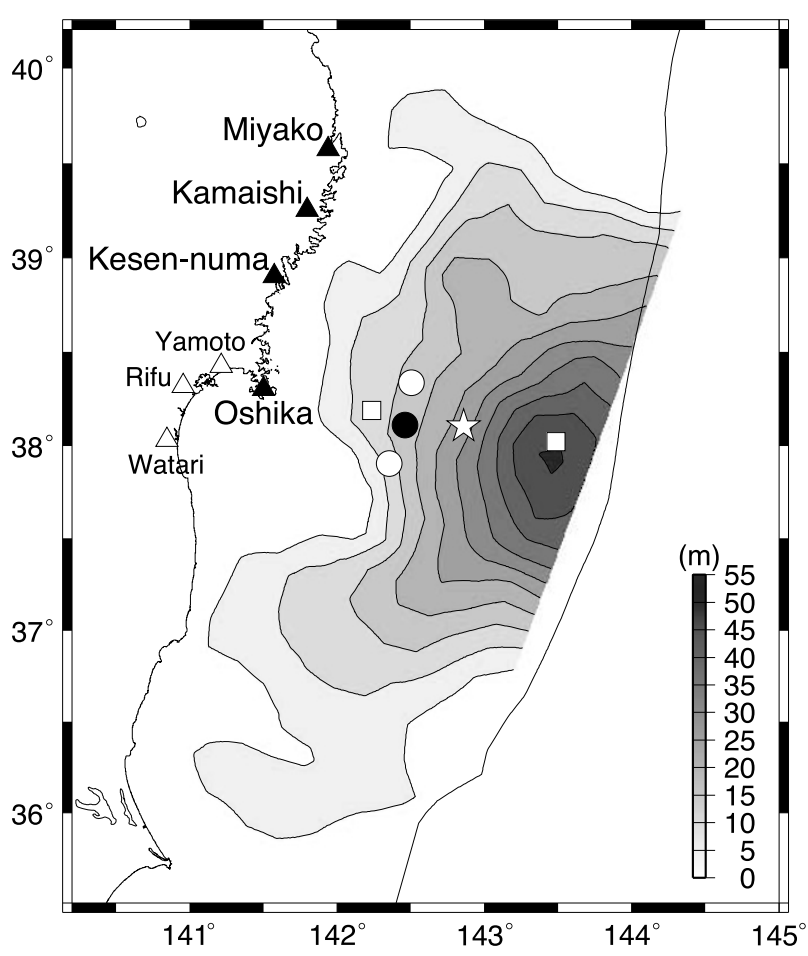

Fig. 1. Location map of the GPS stations used in this study and assumed source locations for model computation, plotted on a contour map of the slip distribution of the 2011 Tohoku-oki earthquake obtained by Yagi and Fukahata (2011). The solid and open triangles represent the location of the GPS stations. The star is the epicenter determined by JMA. Squares and circles are surface projections of the assumed source locations.

processed with a precise point positioning strategy implemented in the GIPSY-OASIS II software (Zumberge et al., 1997). The final (precise) orbits and clocks produced by the Jet Propulsion Laboratory (JPL) are used, and phase ambiguities are resolved.

In Fig. 1, we show a location map of the GPS stations used in this study with an image of the slip distribution (Yagi and Fukahata, 2011) of the 2011 Tohoku-oki earthquake. GPS time series data at the stations (solid triangles in Fig. 1) along the coast from northern Miyagi to southern Iwate prefectures are shown in Fig. 2, where the displacements at Oshika, Kesen-numa, Kamaishi, and Miyako are represented by red, purple, blue, and green, respectively. The origin of time, in Fig. 2, is 14:46:18, local time, on 11th March, 2011. As will be shown later, these GPS stations are suitable for constraining the location of moment release.

The largest displacement in the GEONET was recorded at Oshika. In each station, we can note a very large eastward displacement with a clear time difference from south to north. Interestingly, the large eastward displacement is followed by significant opposite motion. The north-south and vertical displacements are relatively small during the initial $80 \mathrm{~s}$, but the southward displacement at the peak (about $100 \mathrm{~s}$ ) exceeds $2 \mathrm{~m}$ at Kesen-numa and Kamaishi, which is larger than that at Oshika. The southward displacements are also followed by the opposite (northward) displacements. The vertical displacement is largest at Oshika and decreases from south to north.

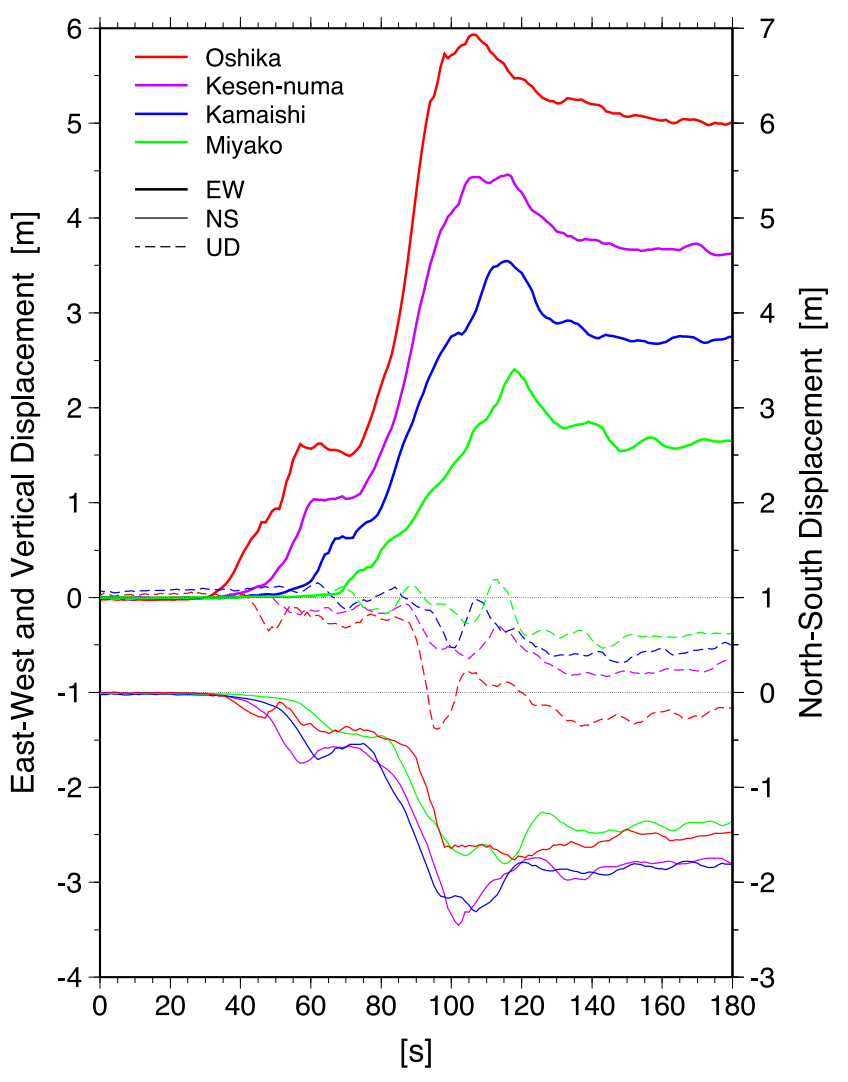

Fig. 2. Observed GPS data with a sampling rate of $1 \mathrm{~Hz}$ at Oshika (red), Kesen-numa (purple), Kamaishi (blue), and Miyako (green), respectively. Three components of displacement, east-west (thick line), north-south (thin line), and vertical (broken line), are shown. For north-south components some offset is given, and so use the scale on the right-hand side. Eastward, northward and upward are taken to be positive. The origin of time is at 14:46:18, local time, on 11th March 2011.

\section{Displacement Due to Dislocation Source}

In an isotropic homogeneous infinite elastic medium, displacement due to a dislocation source, which is generally expressed by a moment tensor, can be analytically evaluated (Aki and Richards, 1980; Matsu'ura, 1996). For example, when the components of a moment tensor $\mathbf{M}(t)$ are zero except for $M_{13}(t)=M_{31}(t)=m(t)$, the displacement of the $i$-th $(i=1,2,3)$ component in Cartesian coordinates at a point $\mathbf{x}$ and a time $t$ is expressed as:

$$
\begin{aligned}
u_{i}(\mathbf{x}, t)= & \frac{3\left(5 \gamma_{i} \gamma_{1} \gamma_{3}-\gamma_{1} \delta_{i 3}-\gamma_{3} \delta_{i 1}\right)}{2 \pi \rho R^{4}} \int_{R / \alpha}^{R / \beta} s m(t-s) d s \\
& +\frac{6 \gamma_{i} \gamma_{1} \gamma_{3}-\gamma_{1} \delta_{i 3}-\gamma_{3} \delta_{i 1}}{2 \pi \rho \alpha^{2} R^{2}} m\left(t-\frac{R}{\alpha}\right) \\
& -\frac{3\left(4 \gamma_{i} \gamma_{1} \gamma_{3}-\gamma_{1} \delta_{i 3}-\gamma_{3} \delta_{i 1}\right)}{4 \pi \rho \beta^{2} R^{2}} m\left(t-\frac{R}{\beta}\right) \\
& +\frac{\gamma_{i} \gamma_{1} \gamma_{3}}{2 \pi \rho \alpha^{3} R} \dot{m}\left(t-\frac{R}{\alpha}\right) \\
& -\frac{2 \gamma_{i} \gamma_{1} \gamma_{3}-\gamma_{1} \delta_{i 3}-\gamma_{3} \delta_{i 1}}{4 \pi \rho \beta^{3} R} \dot{m}\left(t-\frac{R}{\beta}\right),
\end{aligned}
$$

where $R$ and $\gamma_{i}$ are the distance and the direction cosine of the $i$-th $(i=1,2,3)$ component from the source to 


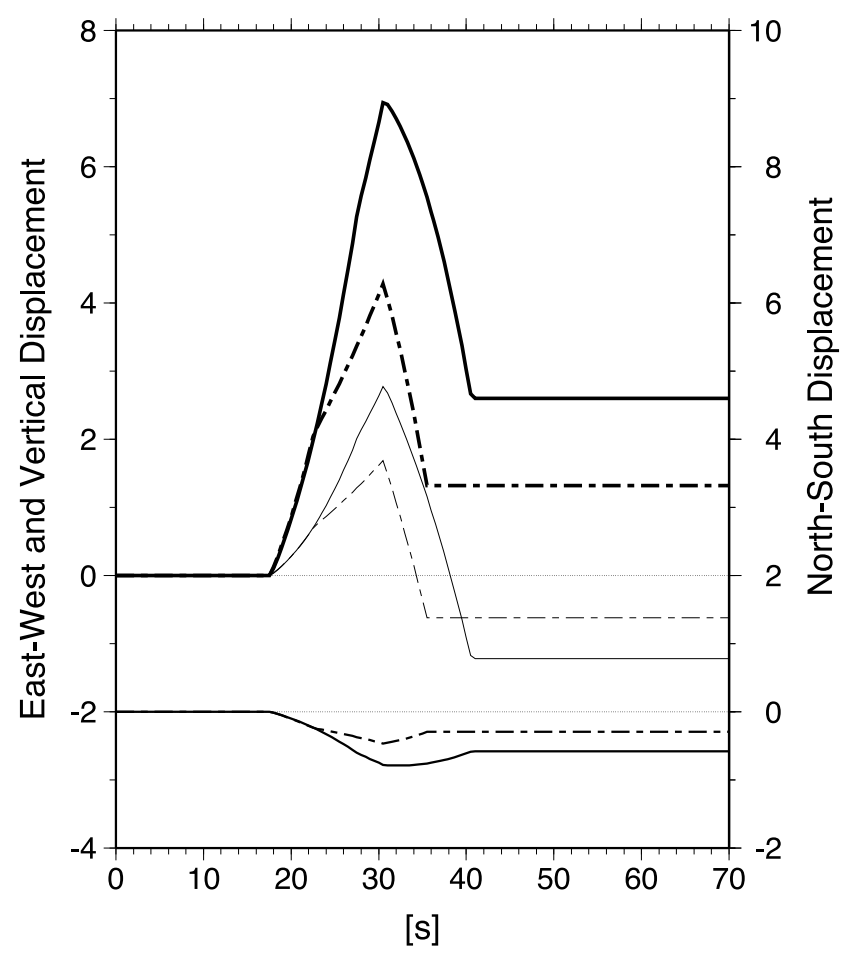

Fig. 3. Computed eastward (thick), northward (middle), and upward (thin) displacements at Oshika due to dislocation sources at the hypocenter. A constant moment release rate is given at the hypocenter for $10 \mathrm{~s}$ (solid line) and $5 \mathrm{~s}$ (chain line) from $t=0$. The magnitude of the moment release rate is suitably normalized.

the observation point. $\alpha, \beta$, and $\rho$ denote the $P$-wave velocity, $S$-wave velocity, and the density of the medium, respectively, and the dot represents the differential with respect to time.

In the following computation, we neglect the far-field terms (fourth and fifth terms in Eq. (1)), because they make a minor contribution to long-period displacement near the source region, as seen in Fig. 2. Static displacements, which are always caused by the near (first term in Eq. (1)) and intermediate terms (second and third terms in Eq. (1)), are clearly dominant in Fig. 2. When we take the ratio of the third (intermediate $S$-wave) term to the fifth (far field $S$ wave) term in Eq. (1) with the values of $R \approx 100 \mathrm{~km}$ and $\beta \approx 4 \mathrm{~km} / \mathrm{s}$, we obtain:

$$
\frac{\text { intermediate } S}{\text { far field } S} \approx \frac{m(t-R / \beta)}{c \dot{m}(t-R / \beta)},
$$

with $c \cong 4 \sim 8 \mathrm{~s}$, which depends on the direction from the source to the observed point. Equation (2) means that the intermediate $S$-wave term is more dominant than the far-field $S$-wave term, if moment release continues longer than several seconds. In addition, the ratio of the second term to the fourth term is about 1.7 times larger than Eq. (2) and the near-field term is also included in the following computation.

As an example (Fig. 3), we computed the displacement at Oshika due to a dislocation source at the hypocenter. In this paper, we use the same fault parameters (strike $200^{\circ}$, dip $\left.12^{\circ}\right)$ and hypocenter location $(38.103 \mathrm{~N}, 142.860 \mathrm{E}$, depth 22 km) as Yagi and Fukahata (2011). We also assume pure dipslip for simplicity, because the 2011 Tohoku-oki earthquake is considered to be basically pure dip-slip (e.g., Yagi and Fukahata, 2011). Some of the inversion analyses (e.g., Lay et al., 2011) show relatively large strike-slip components, but even in their results the strike-slip component is usually small in the area where we assumed point sources in Fig. 1. The $P$-wave velocity, Poisson's ratio, and the density are set at $7.0 \mathrm{~km} / \mathrm{s}, 0.25$, and $3.0 \times 10^{3} \mathrm{~kg} / \mathrm{m}^{3}$, respectively. The moment function $m(t)$ is assumed to linearly increase for $\Delta T$ from $t=0$ (i.e., box-type moment-rate function). Figure 3 shows two cases of $\Delta T, 10 \mathrm{~s}$ (solid line) and $5 \mathrm{~s}$ (chain line).

The onset of displacement is marked by the arrival of a $P$-wave. Displacement usually shows a peak at the arrival of an $S$-wave and significantly moves in the opposite direction after that. This opposite motion is caused by the intermediate $S$-wave term. In some cases, the opposite motion overwhelms the previous displacement (e.g., the vertical component in Fig. 3). When $\Delta T$ is $5 \mathrm{~s}$, in comparison with $\Delta T=10 \mathrm{~s}$, displacement during the initial $5 \mathrm{~s}$ is exactly the same and the final displacement (static displacement) is exactly half.

The setting of the computation of Fig. 3 is very simple: we assumed a homogeneous infinite medium. Hence, we cannot discuss absolute values of moment from a comparison of this computation to observed data. However, the arrival timing of $P$ - and $S$-waves in Fig. 3 should be almost correct to an accuracy of probably less than $10 \%$, from the comparison of travel times with a few representative structure models, such as PREM, CRUST 2.0 (Bassin et al., 2000), and others (e.g., Yagi et al., 2012). The sense of displacement (the position of nodal planes), and the relative amplitude of displacements, are also considered to be basically correct from the analogy of static displacements between an infinite elastic medium and a stratified elastic half-space (Fukahata and Matsu'ura, 2005). Although we cannot discuss short-period displacements because of the neglect of the far-field terms, the travel times of the far-field terms are the same as those of the intermediate terms.

We also have to bear in mind that various waves with different timings and source locations are superimposed on the observed data. In Fig. 4, we exhibit computed displacements at each GPS station caused by dislocation sources at a down-dip point (a), the hypocenter (b), and an up-dip point (c). The location of the sources are denoted by the star and squares in Fig. 1. In the computation of Fig. 4, and the following sections, we take $\Delta T$ to be $10 \mathrm{~s}$ and the other settings are completely the same as in Fig. 3, except source locations which are on the fault plane defined above. Then, the moment given at each source is the same, and so the scale of the vertical axis is different in each diagram depending on the sensitivity of displacement to each source. As can be seen in Fig. 4, it is more difficult to extract source information from vertical displacements, because the final displacement, which is always subsidence, can be cancelled out by the transient displacement, which is usually uplift. On the other hand, in eastward and southward displacements, the static displacements basically steadily accumulate, although the intermediate $S$-wave term considerably modifies the record. 


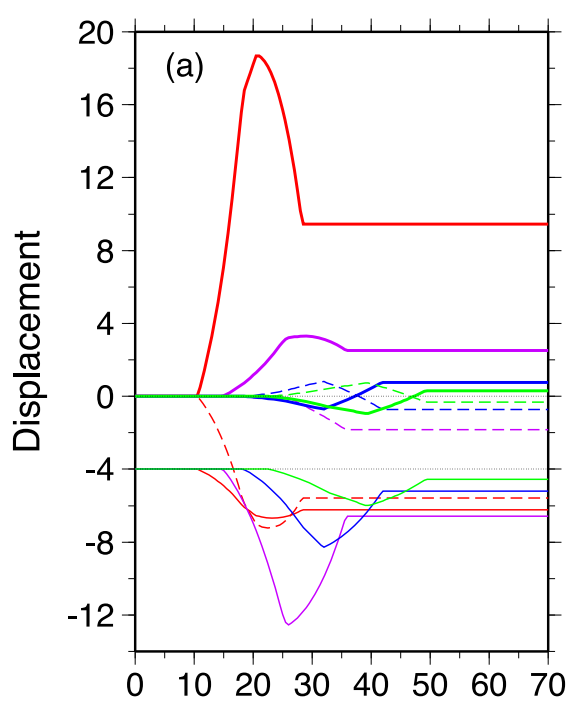

[s]

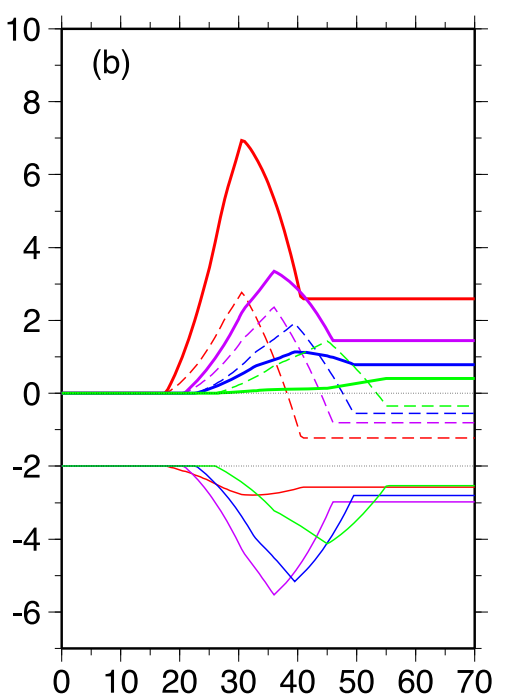

[s]

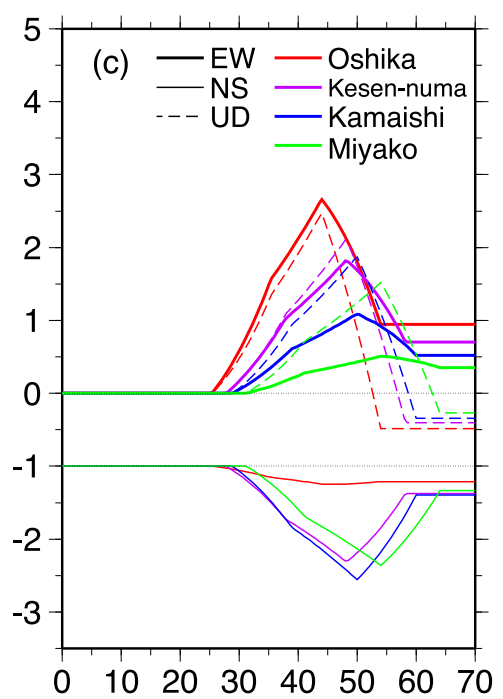

[s]

Fig. 4. Computed displacements at Oshika (red), Kesen-numa (purple), Kamaishi (blue), and Miyako (green) due to dislocation sources at a down-dip point (a), the hypocenter (b), and an up-dip point (c). The source locations are shown by the star and squares in Fig. 1. The same constant moment release rate as in Fig. 3 is given at each source for $10 \mathrm{~s}$ from $t=0$. Thick, thin, and broken, lines represent eastward, northward, and upward displacements, respectively. Some offsets are given for north-south components.

\section{Estimation of Early Rupture Process}

The computed feature of a large eastward displacement followed by a significant opposite motion (Fig. 4) corresponds well to the observed displacement (Fig. 2). As mentioned above, seismic waveform inversion results commonly show a very large slip near the trench around 60 to $80 \mathrm{~s}$. Therefore, the large slip near the trench is considered to be the principal cause of this distinct observed GPS displacement. If we take into account the rigidity contrast along the plate interface (Lay et al., 2011) and the difference of sensitivity of displacement to a dislocation source (Fig. 4), the slip around the hypocenter may also greatly contribute to this distinct GPS displacement. In either case, subsidence and northward motions observed at each station around 2 minutes is consistent with the synthetic computation. As mentioned above, however, displacement at each station is generally caused by dislocation sources at various locations and timings. So, it is not easy to separate the various contributions to displacement except during the early stage.

From Fig. 4, we can see a large eastward displacement at Oshika for every source location. On the other hand, eastward displacements are very small at Kamaishi and Miyako for the down-dip source, and the eastward displacement is again very small at Miyako for the hypocenter source. The observed GPS data (Fig. 2) show that significant displacement at Oshika starts at about $32 \mathrm{~s}$. On the other hand, the eastward displacement at Kamaishi is very small before $50 \mathrm{~s}$ and becomes larger after $60 \mathrm{~s}$. At Miyako, substantial eastward displacement starts around $68 \mathrm{~s}$. If the significant displacement at Oshika is caused by dislocation around the hypocenter, or in the up-dip region, substantial eastward displacement at Kamaishi must be recorded with several seconds delay. Therefore, the first significant moment release must start in the down-dip region.

It should also be noted that the computed displacement of Fig. 4(a) is clearly different from the observed data at Oshika in the vertical component, which is the most sensitive to the change of the source location in the acrossarc direction. When we compute the displacement for a dislocation source shown by the solid circle in Fig. 1, the vertical displacement at Oshika is also consistent with the observed data (Fig. 5); it is very small during the initial $10 \mathrm{~s}$ after the arrival of the $P$-wave. If the source moves to the north-south direction, as represented by the open circles in Fig. 1, then the north-south displacement component at Oshika is significantly affected (Fig. 6). For the northern source (chain line), the sense of north-south displacement is reversed, and for the southern source (solid line), the northsouth displacement attains a similar amplitude to the eastwest displacement. In determining the location of the first significant moment release, the assumption of a uniform dip angle for the fault plane might be too simple. If the dip angle around the solid circle in Fig. 1 is larger by a few degrees than the assumed 12 degrees, then the point with almost no motion in the vertical displacement before the arrival of the $S$-wave (Fig. 5) moves eastward several kilometers, which corresponds to the radius of the solid circle in Fig. 1. In short, the first significant moment release that is responsible to the onset of the displacement at Oshika should occur around the solid circle in Fig. 1 from about 20 s. Before about $20 \mathrm{~s}$, the moment release of the 2011 Tohoku-oki earthquake must have been so small that any substantial displacements were not observed at GPS stations on land.

The eastward displacement at Kamaishi gradually grows from $50 \mathrm{~s}$ to $60 \mathrm{~s}$ (Fig. 2). The eastward displacement of this period may be caused by the dislocation source around the hypocenter, but it is also possible that the same source responsible for the significant displacement at Oshika from 32 s causes this movement. As shown in Fig. 5, static eastward displacement at Kamaishi amounts to about $15 \%$ of that at Oshika. However, the displacement after $60 \mathrm{~s}$ at 


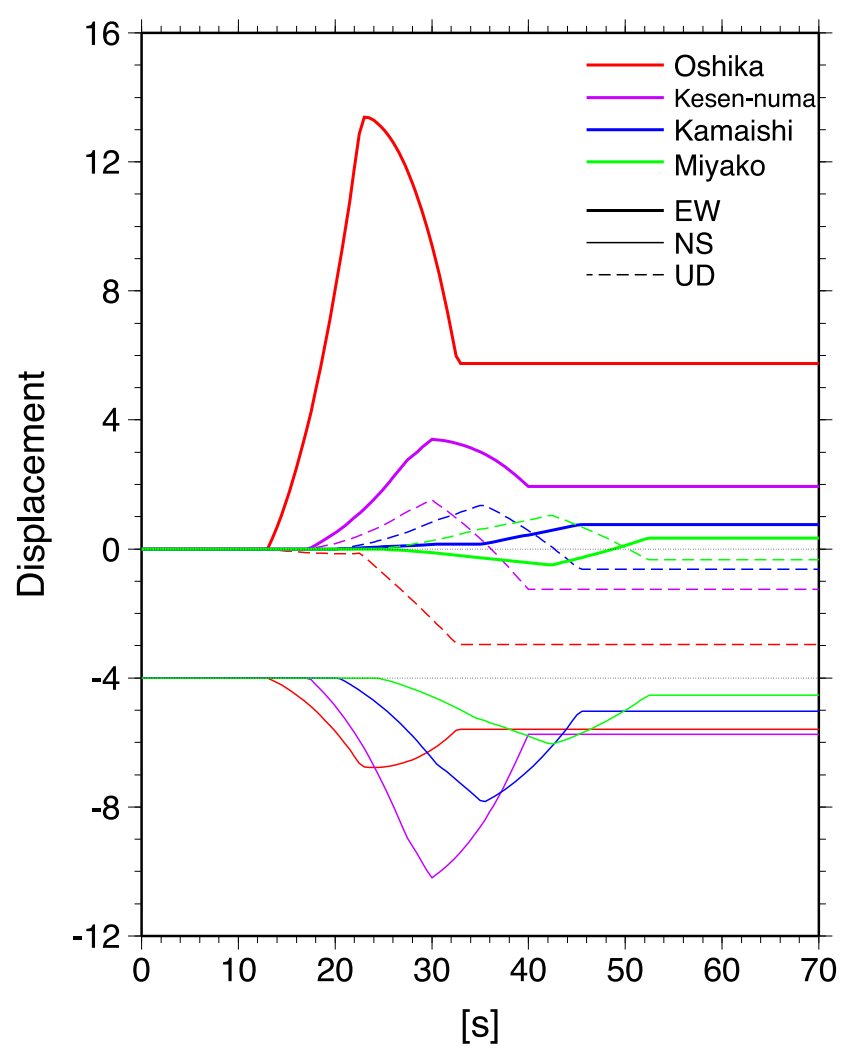

Fig. 5. Computed displacements at Oshika (red), Kesen-numa (purple), Kamaishi (blue), and Miyako (green) due to a dislocation source at the solid circle shown in Fig. 1. The setting of the computation is the same as in Fig. 4 except for the source location. Thick, thin, and broken, lines represent eastward, northward, and upward displacements, respectively. For the north-south component some offset is given.

Kamaishi is much larger, and so the source location must be different. If the source locates around the up-dip point, as shown in Fig. 4(c), the eastward displacement at Kamaishi must be followed by that at Miyako with a few seconds delay. Therefore, the onset of substantial eastward displacement at Kamaishi is not due to the source near the trench, but maybe around the hypocenter. In short, taking the travel time shown in Fig. 4(b) into account, the moment release around the hypocenter started from about $25 \mathrm{~s}$ if it caused the eastward displacement from $50 \mathrm{~s}$ at Kamaishi, and started from about $35 \mathrm{~s}$ if it caused the eastward displacement from $60 \mathrm{~s}$. However, it is not easy to completely exclude the possibility that this significant eastward motion at Kamaishi was caused by a down-dip source to the north of the northern open circle in Fig. 1. Then, northward motion must transiently arrive at Oshika, but such motion can be cancelled out by the southward displacement due to other sources to the south.

Because substantial displacement starts around $68 \mathrm{~s}$ at Miyako, significant moment release in the up-dip region is considered to start around $35 \mathrm{~s}$ (Fig. 4(c)). Again, this estimate is the earliest timing. In fact, significant moment release must occur in the region to the north of the hypocenter and the assumed near-trench source (open square in Fig. 1) as well, because a clear westward motion after peaking in the eastward displacement (about $120 \mathrm{~s}$ ) is observed even in Miyako (Figs. 2 and 4(c)).

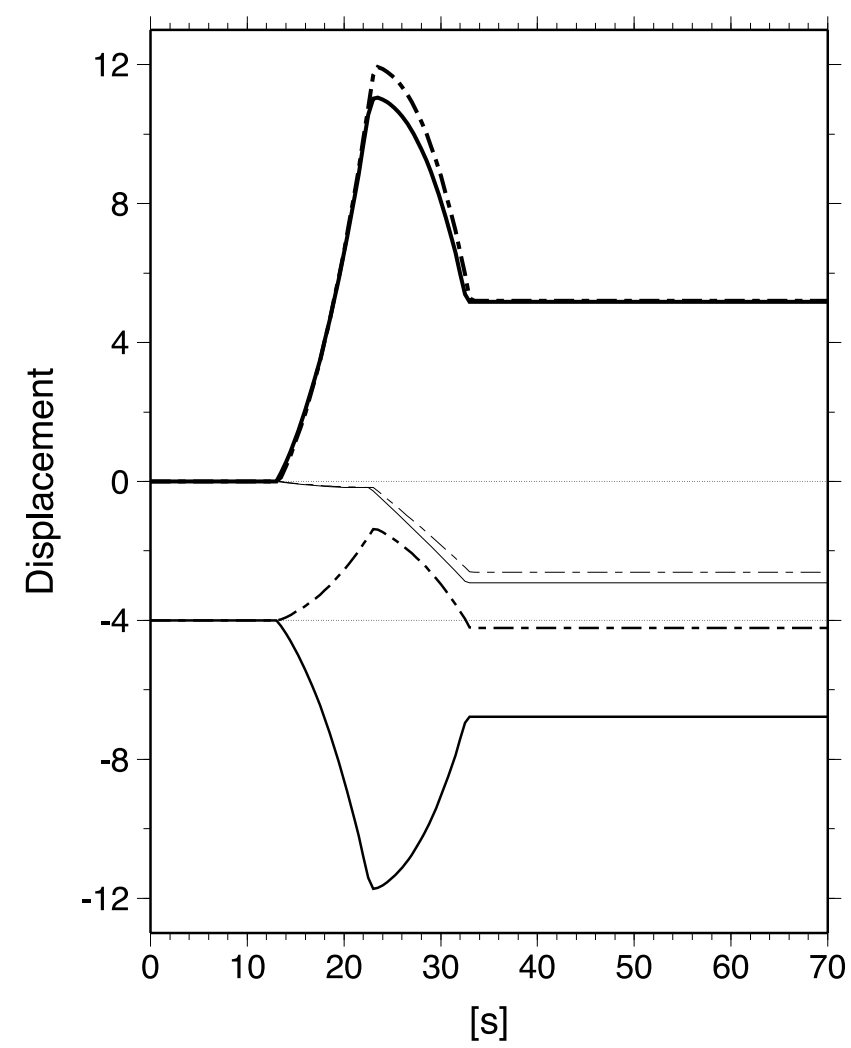

Fig. 6. Computed displacements at Oshika due to a dislocation source at the southern-open (solid line) and northern-open (chain line) circles shown in Fig. 1. The setting of the computation is the same as in Fig. 4 except for the source location. Thick, middle, and thin, lines correspond to eastward, northward, and upward displacements, respectively. Some offset is given for the north-south component.

\section{Discussion and Conclusions}

From the comparison of observed 1-Hz GPS data with simple forward computation, we conclude that the first significant moment release in the 2011 Tohoku-oki earthquake started around the solid circle in Fig. 1 from about $20 \mathrm{~s}$, and that significant moment release around the hypocenter, and in the up-dip region, started from about $25 \mathrm{~s}$ and $35 \mathrm{~s}$ at the earliest, respectively. So far, we have shown the GPS data only at a limited number of stations for simplicity, but data from other stations are also consistent with the above conclusions. In Fig. 7(a), we show observed displacements at Yamoto (purple), Rifu (blue), and Watari (green) together with Oshika (red). Figure 7(b) shows the computed displacements at these stations due to the dislocation source at the solid circle in Fig. 1. We can see that the onset timing of significant eastward displacements, as well as the sense and relative amplitude of north-south displacements, are well consistent in Figs. 7(a) and (b). In this paper, we consider a simple model by assuming a point source, a box-type moment rate function, pure dip-slip, simple plate geometry, an infinite homogeneous medium, and neglect of far-field terms. With such a simple model, the observed $1-\mathrm{Hz}$ GPS data in the early stage of this earthquake can be well explained.

As mentioned above, the results of seismic waveform inversion commonly show a shoulder in the moment-rate function at around $35 \mathrm{~s}$. The observed eastward displace- 


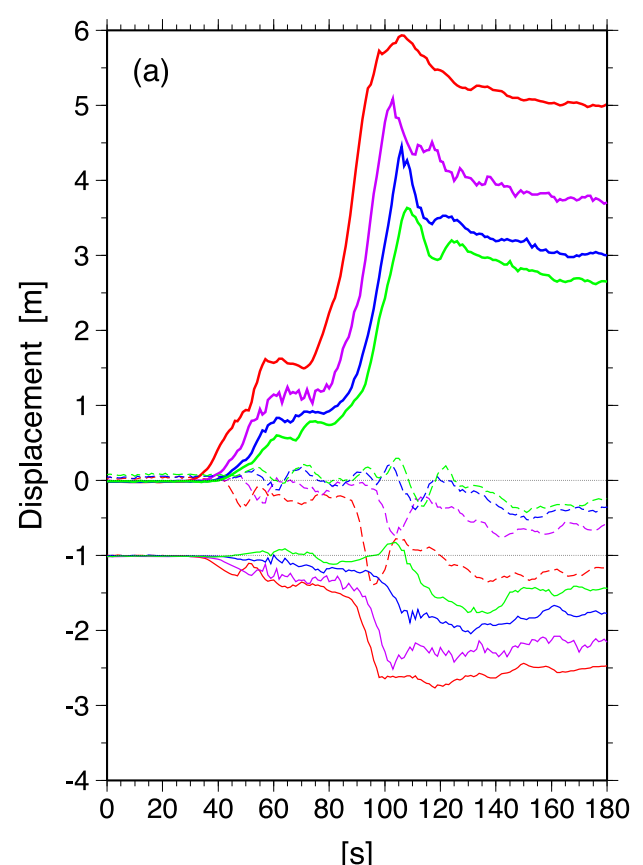

[s]

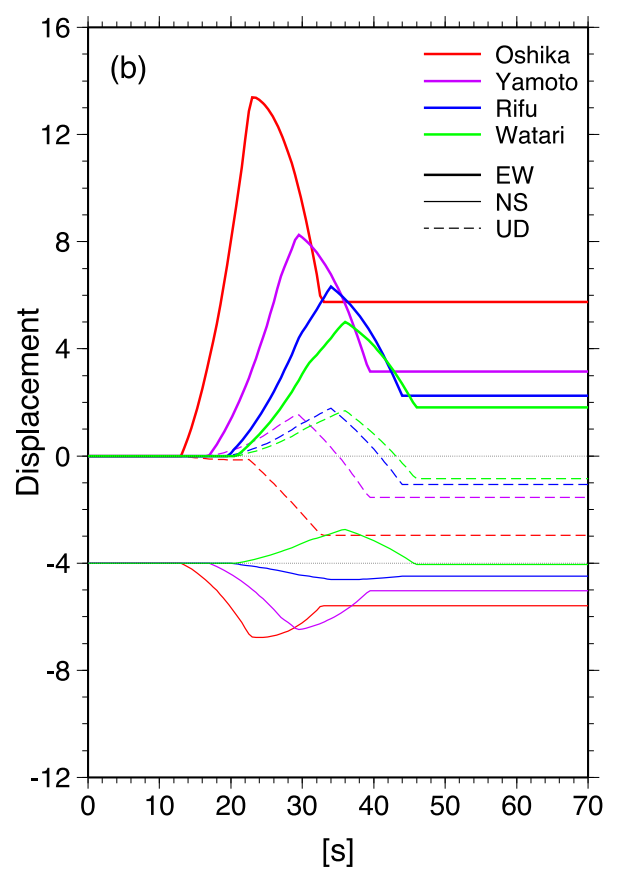

[s]

Fig. 7. Observed (a), and computed (b), displacements at Oshika (red), Yamoto (purple), Rifu (blue), and Watari (green). The same dislocation source as in Fig. 5 is used for the computation. Thick, thin, and broken, lines represent eastward, northward, and upward displacements, respectively. Some offset is given for the north-south component.

ments at Oshika, Kesen-numa, and the stations in Fig. 7, also have clear shoulders approximately from $55 \mathrm{~s}$ to 70 s (Figs. 2 and 7(a)). As shown in Figs. 5 and 7(b), these observed data can be well explained by a cessation of significant moment release roughly at $35 \mathrm{~s}$. That is to say, in the 2011 Tohoku-oki earthquake, significant moment release first occurred in the down-dip region approximately from $20 \mathrm{~s}$ to $35 \mathrm{~s}$, which is well consistent with the inversion result of Yagi and Fukahata (2011). The shoulder of the moment-rate function is considered to be caused in this way. On the other hand, significant moment release near the trench started after $35 \mathrm{~s}$, which is clearly later than their result. A large moment release, first in the down-dip region, followed by a large slip near the trench, well corresponds to the inversion result of Ide et al. (2011), but the timing of the transition is later and the location of large down-dip slip is apparently too close to the coast in their result.

Analyses of high-rate GPS data are relatively new (e.g., Ji et al., 2004; Miyazaki et al., 2004; Ammon et al., 2011; Yue and Lay, 2011; Ohta et al., 2012; Wright et al., 2012). However, as shown by this study, high-rate GPS data contain rich information about the rupture process of earthquakes. For example, the southward displacement at Watari after $100 \mathrm{~s}$ is considered to correspond to the rupture propagation southward in the later stage of the 2011 Tohoku-oki earthquake (e.g., Suzuki et al., 2011; Koketsu et al., 2011; Yagi and Fukahata, 2011; Yue and Lay, 2011). Fast downward displacement around 90 to $95 \mathrm{~s}$ at Oshika is accompanied by fast southward and eastward displacements. This motion may be caused by a dislocation source to the south of the down-dip square and to the west of the southern open circle in Fig. 1. To fully extract the rich information from high-rate GPS data is an interesting challenge.
Acknowledgments. The figures were created with the GMT software by Paul Wessel and Walter H. F. Smith (Wessel and Smith, 1998). We thank anonymous reviewers for useful comments. This study was supported by Grant-in-Aid for Scientific Research No. 21540428 and 24540450 of the Japan Ministry of Education, Culture, Sports, Science and Technology to YF and YY.

\section{References}

Aki, K. and P. G. Richards, Quantitative Seismology, Volume I, 557 pp, W. H. Freeman and Company, New York, 1980.

Ammon, C. J., T. Lay, H. Kanamori, and M. Cleveland, A rupture model of the 2011 off the Pacific coast of Tohoku Earthquake, Earth Planets Space, 63, 693-696, 2011.

Asano, K. and T. Iwata, Source model for strong ground motion generation in the frequency range $0.1-10 \mathrm{~Hz}$ during the 2011 Tohoku earthquake, Earth Planets Space, 64, this issue, 1111-1123, 2012.

Bassin, C., G. Laske, and G. Masters, The current limits of resolution for surface wave tomography in North America, Eos Trans. AGU, 81, 897, 2000.

Fukahata, Y. and M. Matsu'ura, General expressions for internal deformation fields due to a dislocation source in a multilayered elastic halfspace, Geophys. J. Int., 161, 507-521, 2005.

Hayes, G. P., Rapid source characterization of the $2011 M_{\mathrm{w}} 9.0$ off the Pacific coast of Tohoku Earthquake, Earth Planets Space, 63, 529-534, 2011.

Honda, R., Y. Yukutake, H. Ito, M. Harada, T. Aketagawa, A. Yoshida, S. Sakai, S. Nakagawa, N. Hirata, K. Obara, and H. Kimura, A complex rupture image of the 2011 off the Pacific coast of Tohoku Earthquake revealed by the MeSO-net, Earth Planets Space, 63, 583-588, 2011.

Ide, S., A. Baltay, and G. C. Beroza, Shallow dynamic overshoot and energetic deep rupture in the $2011 \mathrm{Mw} 9.0$ Tohoku-oki earthquake, Science, 332, 1426-1429, 2011.

Ji, C., K. M. Larson, Y. Tan, K. W. Hudnut, and K. Choi, Slip history of the 2003 San Simeon earthquake constrained by combining 1-Hz GPS, strong motion, and teleseismic data, Geophys. Res. Lett., 31, L17608, doi:10.1029/2004GL020448, 2004.

Koketsu, K., Y. Yokota, N. Nishimura, Y. Yagi, S. Miyazaki, K. Satake, Y. Fujii, H. Miyake, S. Sakai, Y. Yamanaka, and T. Okada, A unified source model for the 2011 Tohoku earthquake, Earth Planet. Sci. Lett., 310, 480-487, 2011.

Lay, T., C. J. Ammon, H. Kanamori, L. Xue, and M. J. Kim, Possible large near-trench slip during the $2011 M_{\mathrm{w}} 9.0$ off the Pacific coast of Tohoku 
Earthquake, Earth Planets Space, 63, 687-692, 2011.

Lee, S. J., B. S. Huang, M. Ando, H. C. Chiu, and J. H. Wang, Evidence of large scale repeating slip during the 2011 Tohoku-Oki earthquake, Geophys. Res. Lett., 38, L19306, doi:10.1029/2011GL049580, 2011.

Matsu'ura, M., Deformation and fracture, in Earth and Planetary Science 6: Earth's Continuum Mechanics, 175-237, Iwanami Shoten, 1996 (in Japanese).

Miyazaki, S., K. M. Larson, K. Choi, K. Hikima, K. Koketsu, P. Bodin, J. Haase, G. Emore, and A. Yamagiwa, Modeling the rupture process of the 2003 September 25 Tokachi-Oki (Hokkaido) earthquake using 1 Hz GPS, Geophys. Res. Lett., 31, L21603, doi:10.1029/2004GL021457, 2004.

Ohta, Y., T. Kobayashi, H. Tsushima, S. Miura, R. Hino, T. Takasu, H. Fujimoto, T. Iinuma, K. Tachibana, T. Demachi, T. Sato, M. Ohzono, and N. Umino, Quasi real-time fault model estimation for near-field tsunami forecasting based on RTK-GPS analysis: Application to the 2011 Tohoku-Oki earthquake ( $M_{\mathrm{w}}$ 9.0), J. Geophys. Res., 117, B02311, doi:10.1029/2011JB008750, 2012.

Shao, G., X. Li, C. Ji, and T. Maeda, Focal mechanism and slip history of the $2011 M_{\mathrm{w}} 9.1$ off the Pacific coast of Tohoku Earthquake, constrained with teleseismic body and surface waves, Earth Planets Space, 63, 559$564,2011$.

Suzuki, W., S. Aoi, H. Sekiguchi, and T. Kunugi, Rupture process of the 2011 Tohoku-Oki mega-thrust earthquake (M9.0) inverted from strong-motion data, Geophys. Res. Lett., 38, L00G16, doi:10.1029/2011GL049136, 2011.

Wessel, P. and W. H. F. Smith, New, improved version of generic mapping tools released, Eos Trans. AGU, 79, 579, 1998.

Wright, T. J., N. Houlié, M. Hildyard, and T. Iwabuchi, Real-time, reliable magnitudes for large earthquakes from $1 \mathrm{~Hz}$ GPS precise point positioning: the 2011 Tohoku-Oki (Japan) earthquake, Geophys. Res. Lett., 39, L12302, doi:10.1029/2012GL051894, 2012.

Yagi, Y. and Y. Fukahata, Rupture process of the 2011 Tohoku-oki earthquake and absolute elastic strain release, Geophys. Res. Lett., 38, L19307, doi:10.1029/2011GL048701, 2011.

Yagi, Y., A. Nakao, and A. Kasahara, Smooth and rapid slip near the Japan Trench during the 2011 Tohoku-oki earthquake revealed by a hybrid back-projection method, Earth Planet. Sci. Lett., 355-356, 94101, 2012.

Yoshida, K., K. Miyakoshi, and K. Irikura, Source process of the 2011 off the Pacific coast of Tohoku Earthquake inferred from waveform inversion with long-period strong-motion records, Earth Planets Space, 63, 577-582, 2011.

Yoshida, Y., H. Ueno, D. Muto, and S. Aoki, Source process of the 2011 off the Pacific coast of Tohoku Earthquake with the combination of teleseismic and strong motion data, Earth Planets Space, 63, 565-569, 2011.

Yue, H. and T. Lay, Inversion of high-rate (1 sps) GPS data for rupture process of the 11 March 2011 Tohoku earthquake (Mw 9.1), Geophys. Res. Lett., 38, L00G09, doi:10.1029/2011GL048700, 2011.

Zumberge, J. F., M. B. Heflin, D. C. Jefferson, M. M. Watkins, and F. H. Webb, Precise point positioning for the efficient and robust analysis of GPS data from large networks, J. Geophys. Res., 102, 5005-5017, 1997.

Y. Fukahata (e-mail: fukahata@rcep.dpri.kyoto-u.ac.jp), Y. Yagi, and S. Miyazaki 